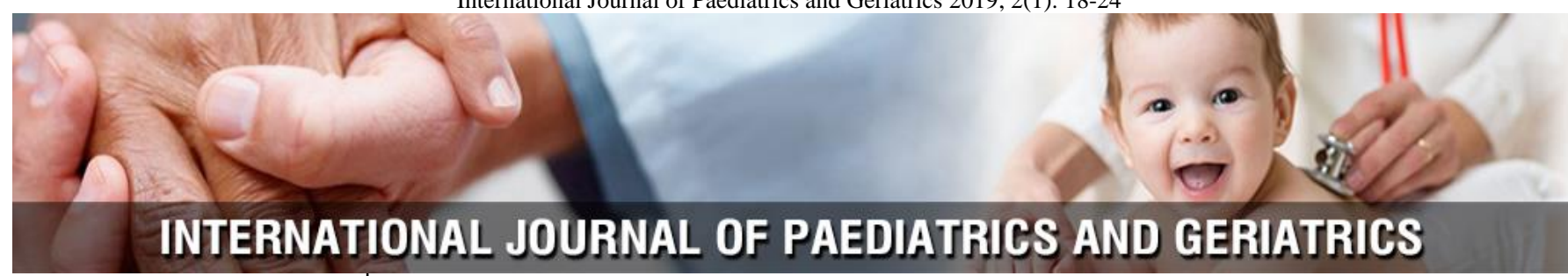

P-ISSN: 2664-3685

E-ISSN: 2664-3693

www.paediatricjournal.com

IJPG 2019; 2(1): 18-24

Received: 26-11-2018

Accepted: 29-12-2018

Salwa Ali Marzouk

Assistant Professor of pediatrics nursing, Faculty of

Nursing, Assiut University

Egypt

Amal Abdrbou Hussien Lecturer of pediatrics nursing, Faculty of Nursing, Assiut

University Egypt

Safwat Mohamed Abdel Aziz Lecturer of pediatrics \& neonatology, Faculty of Medicine, Assiut University Egypt
Corresponding Author:

Salwa Ali Marzouk

Assistant Professor of

pediatrics nursing, Faculty of

Nursing, Assiut University

Egypt

\section{Effectiveness of cycled lighting in neonatal intensive care unit on weight and cardiorespiratory function in preterm infants}

\author{
Salwa Ali Marzouk, Amal Abdrbou Hussien and Safwat Mohamed Abdel Aziz
}

DOI: https://doi.org/10.33545/26643685.2019.v2.i1a.23

\begin{abstract}
Hospitalization in neonatal intensive care units predisposes preterm neonates to negative stimuli such as continuous 24-hourlighting. Potential benefits and harms of different lighting in neonatal units have not been quantified. Therefore, this study aimed to evaluate the benefits of a cycled lighting (light/dark cycle) in the Neonatal Intensive Care Unit on weight and cardiorespiratory function in premature infants.
\end{abstract}

A Randomized Clinical Trial: Performed on preterm neonates who hospitalized in NICU at Assiut University Children Hospital, Egypt. From admission to discharge, neonates were randomly exposed to cyclical lighting (study) or continuous lighting (control) groups, twenty in each one. A structured questionnaire was used to collect data and included personal data, weight and cardiorespiratory assessment.

The Results: Of the study revealed that mean of neonates' weight in the study group was significantly greater than control group (P. 0.017). Mean pulse rates at 10 days and at discharge were significantly more stable in the study group compared to control group $(p<0.002 \& p<0.028)$. Moreover, the mean $\mathrm{O}_{2}$ saturation is significantly better in study group than control $(P<0.045)$.

Conclusions: Cycled lighting is an effective strategy to improve premature neonates' weight and cardiorespiratory function.

Recommendations: $T$ Nurses should be aware of the importance of applying the Cycled lighting at NICU to improve preterm neonates' health.

Keywords: Cycled lighting, weight, cardiorespiratory function, and preterm infants.

\section{Introduction}

During the last two decades, the number of preterm neonates has increased mainly due to an increase in the number of preterm deliveries. Around 310 million neonates are born annually in the world, while $15 \%$ of them are premature (W.H.O., 2017). Premature neonates need life support services and hence are hospitalized in Neonatal Intensive Care Unit (NICU) (Klock and Erdmann 2012) ${ }^{[8]}$. Evidence reports high rates of prolonged hospitalization in developing countries (Martin, et al., 2014) with a mean NICU stay of as high as 33 days (Alizadeh, et al., 2013) ${ }^{[1]}$.

Although neonatal intensive care units provide highly specialized medical care, they do not necessarily offer an ideal environment for the development of newborn infants. The physical environment (light, temperature, sound, radiations) in the NICU is a critical issue, which can affect the normal development of a newborn infant (Hunt, 2011) ${ }^{[6]}$.

There is a huge difference between intrauterine environment and the NICU environment. The pregnant woman is exposed to variable intensities of light and sound, and generally to lower levels at night. Some of the light and sound reaches the fetus within the womb and induces circadian rhythms. 'Circadian' is a term used to describe biological processes that recur naturally on a 24-hour basis. After birth, preterm infants are cared for in an environment that has no planned light-dark cycles and no other circadian entraining signals (Morag and Olsson 2016) ${ }^{[14]}$.

One of the main concerns about neonates, who are hospitalized in the NICUs, is their early exposure to intense and unpredictable lighting. In these units, neonates are almost constantly exposed to continuous lighting which have been associated with adverse clinical outcomes: less weight gain, behavioral and sleep disturbances, in addition to stress in preterm. (Peng, et al., 2009) ${ }^{[17]}$. 
Using artificial entraining signals in the nursery, that is, CL may prevent these Disturbances and may help to promote growth while preventing other morbidities. CL has the potential to promote circadian rhythms that confer health benefits, including hormonal regulation, activity-rest cycles and vital sign regulation, with the potential of promoting infant growth (Verklan and Walden, 2014) ${ }^{[21]}$. Therefore, a controlled intervention (cycled lighting) may have systematically favored physiological stability and reduced motor activity level in the studies with a continuous bright light comparison group. In contrast, continuous light or dark conditions delay the onset of circadian behavioral rhythms and lead to reduced weight gain in preterm infants (Morag and Olsson 2016) [14].

Compared to other healthcare professionals, nurses are more actively involved in providing care to neonates. Therefore, nurses should control lighting in the NICU to promote the environmental adaptation of preterm infants and avoid unsuitable responses indicative of stress. In this way, one of the strategies, which can be aimed at lighting control, is to create artificial day and night. This strategy is noninvasive, incurs no cost, and can be used as a basis for organizing the delivery of other care services.

Significant of the study Previous studies with significant improvement in physiological stability and motor activity levels in premature infants compared a controlled lighting intervention to continuous bright light, which is known to be related to physiological instability and increased motor activity level in premature infants (Rivkees, et al., 2004) ${ }^{[19]}$. One study also, comparing the effects of continuous and cyclical lightings among preterm neonates indicated that effectiveness of cyclical lightings in improving weight gain and shortening hospital stay (Esmaeilizadeh, et al., 2016) ${ }^{[3]}$. While a clinical study showed that cycled lighting had no significant effects on weight gain (Guyer $e t$ al., 2012) ${ }^{[5]}$. Due to the contradictory results of previous studies, this study conduct to confirm the best way of lighting in NICUs.

\section{Aim of this study}

The study aimed to evaluate the benefits of a cycled lighting in the NICU (light/dark cycle) on weight and cardiorespiratory function in premature infants.

This was done through the following:

1. Evaluate the effect of cycled lighting versus continuous light on weight in preterm infants.

2. Investigate the effect of the intervention (cycled lighting) on cardiorespiratory function in premature infants.

3. Assess the effect of the intervention (cycled lighting) on hospital stay in premature infants.

\section{The hypothesis of the study}

H1: Infants with cycled light have greater weight than those who exposed to continuous light.

H2: Infants with cycled light possible have better cardiorespiratory function than those who exposed to continuous light.

H3: Duration of hospital stay in cycled lighting group is shorter than continuous light group.

\section{Subjects and Methods}

\section{Research Design}

Randomized clinical trial was used in this study.

\section{Setting}

This study was conducted in Neonatal Intensive Care Unit (NICU) at Assiut University Children Hospital.

\section{Sample}

Sample size was calculated based on previous studies (the Lee and Bang (2011) ${ }^{[10]}$. study for which physiological stability was evaluated and a group size of 16 subjects per group was calculated for a total sample of 32. Also, the experimental study by Rivkees $e t$ al. (2004) ${ }^{[19]}$. Which explored cycled lighting, the size of the sample was 38 participants (19 per group). A purposive sample of 40 neonates (both sex) admitted to NICU included in the study. They assigned to two groups; cycled lighting (study group) or continuous light (control group). Neonates selected based on the inclusion and exclusion criteria. Inclusion criteria included infants were $\geq 32$ weeks of gestational age (according maternal last menstrual period, or the Ballard scores), delivering by vaginal or cesarean section delivery, hospitalized in the NICU for at least for 10 days. Exclusion criteria included; Neonates with congenital anomalies, who requiring a long period (more than $12 \mathrm{~h}$ ) of continuous lighting for frequent treatment interventions, were receiving medications like catecholamine that could influence cardiorespiratory function stability, had obtained an Apgar score less than 6 at 5 minutes of life or who died before discharge.

\section{Tools}

A structured questionnaire was used to collect data pertinent to the study and included the following parts

\section{Part one}

\section{Personal Data}

It included sex, gestational age, age on admission, date of admission, date of discharge, diagnosis and mode of delivery. The data for this part was extracted from infants' medical records. Length of hospital stay was calculate as the time from admission until day of discharge home.

\section{Part Two}

\section{Weight gain assessment}

Studied infants were weighed each morning, using a digital weighing scale (SECA, Germany) by the nursing staff in charge of each infant. The infants' weight on admission (as baseline), on $10^{\text {th }}$ day and on discharge documented in a standard format. All infants in both groups weighed naked.

\section{Part Three}

Assessment of cardiorespiratory function to evaluate heart rate, respiratory rate, and $\mathrm{O} 2$ saturation levels. Observation for the 3 parameters over the 24-hour, daytime, and nighttime periods were done. The Coefficient of Variation (CV) (standard deviation divided by the mean) and the means were also calculated for each of them. The CV indicates the variability of the physiological parameter. This makes it possible to identify a physiological parameter that varies considerably, but whose mean is situated within the normal range.

\section{Reliability and validity of the tools}

Tools submitted to a panel of three experts in the field of Pediatric Nursing and Medicine to test the content validity. No Modifications were carried out according to the panel judgment on the clarity of the sentences and appropriateness of the content. The content validity was $94 \%$. Reliability analysis was conducted to investigate the instrument internal consistency, which used in the study; Cronbach alpha coefficients was calculated to examine the measurement reliability with multipoint items and its result was $R=0.77$.

\section{Operational definitions Cycled Light (CL)}

Is define as lighting that follows a day-night cycle to promote the establishment of a circadian rhythm in preterm infants.

\section{Pilot study}

A total $10 \%$ of the sample (4) were included in the pilot study in order to assess the feasibility and clarity of the tool. Neonates included in the pilot study excluded from the study sample.

\section{Field of the work}

\section{Assessment phase}

In this phase, data was collected to cover a period of 6 months from the beginning of May 2017 to end of October 2017 in the 
Neonatal Intensive Care Unit at Assiut University Children Hospital.

\section{Implementation phase}

In this phase, the selected neonates were assigned into two groups (20 for each). At the begining, researchers provided all nurses instructions about the study intervention in three separate sessions (the morning, evening, and night shift). The first selected group was the control group. No protocol or single definition is available for time cycles or for maximal and minimal lux lighting used for cycled lighting, which usually provided in a 12-hours day/12-hours night pattern, similar to changes in natural light. A time of transition between day and night occurs at the change of nursing shifts (7 am and $7 \mathrm{pm}$ ). Bright fluorescent strip lights that remained permanently lighted on the control group. During the daytime, the environment was identical in the two groups. At nighttime $(7 \mathrm{pm})$ in the study group infants, the cycled lighting intervention achieved by placing dark pads over the infant's eyes and then maintained until $7 \mathrm{am}$. The intervention continued at least for 10 days or up to discharge. The nurses of the study setting implemented cycled lighting. To ensure that all staff were compliant with the types of lighting regimen assigned to the infants, twice a day, one of the researchers checked that the lighting protocol for each infant was adhered to.

\section{Evaluation Phase}

For both groups, weight and cardiorespiratory function evaluated on admission, after 10 days and on discharge.

\section{Ethical Considerations}

An official permission was taken from the authoritative personal in the hospital. The researchers introduced themselves to the neonate's parent whom their baby met the inclusion criteria and informed them about the aim of the current study and written assent obtained from them. Confidentiality and anonymity of them assured through coding the data.

\section{Statistical analysis}

Data was coded and transformed into a specially designed format suitable for computer feeding. All entered data verified for any errors. Data were analyzed using statistical package for social sciences (SPSS) version 20 windows and presented in tables and graphs. Frequencies analysis performed and Chi-square test were used in comparison between two related groups having qualitative data. In addition, repeated measures Fisher Exact test, MannWhitney test, mean and standard deviations were computed. An alpha level of 0.05 was used to assess significant differences.

\section{Results}

Table 1: Personal characteristics distribution of the studied infants in two groups.

\begin{tabular}{|c|c|c|c|}
\hline Item & Study $(n=20)$ & Control $(n=20)$ & P-value \\
\hline \multicolumn{3}{|c|}{ Sex } & \multirow{3}{*}{0.451} \\
\hline Male & $14(70.0 \%)$ & $17(85.0 \%)$ & \\
\hline Female & $6(30.0 \%)$ & $3(15.0 \%)$ & \\
\hline \multicolumn{3}{|c|}{ Mode of delivery } & \multirow{3}{*}{0.723} \\
\hline Cesarean Section & $15(75.0 \%)$ & $14(70.0 \%)$ & \\
\hline Normal Vaginal Delivery & $5(25.0 \%)$ & $6(30.0 \%)$ & \\
\hline \multicolumn{3}{|c|}{ Gestational age: (weeks) } & \multirow{3}{*}{0.780} \\
\hline Mean \pm SD & $33.70 \pm 1.45$ & $33.60 \pm 1.54$ & \\
\hline Range & $32.0-36.0$ & $32.0-36.0$ & \\
\hline
\end{tabular}

The study sample included 40 neonates admitted in Neonatal Intensive Care Unit (NICU) at Assiut University Children Hospital. Table (1) denotes personal characteristics of the studied neonates in the study (infants exposed to cycled lighting) and control (infants exposed to continuous lighting) groups. The mean gestational age on admission of the two groups was thirty-three weeks. The majority of the study and control groups were male (70.0\% and $85.0 \%$, respectively). Furthermore, there was a similarity between the two groups regarding mode of delivery mission.

Table 2: Mean and Median duration of feeding routes among the studied infants at both groups.

\begin{tabular}{|c|c|c|c|}
\hline & Study (n= 20) & Control (n= 20) & \multirow{2}{*}{ P-value } \\
\cline { 2 - 3 } & Gavage feeding & \multirow{2}{*}{$0.001^{*}$} \\
\hline Mean \pm SD & $2.25 \pm 0.50$ & $5.83 \pm 5.88$ & \\
\hline Median (Range) & $2.0(2.0-3.0)$ & $2.5(2.0-16.0)$ & \multirow{2}{*}{$0.050^{*}$} \\
\hline Men \pm SD & $19.62 \pm 12.29$ & $9.71 \pm 9.51$ & \\
\hline Median (Range) & $23.5(1.0-35.0)$ & $7.0(1.0-30.0)$ & \\
\hline
\end{tabular}

Table 2 illustrates that the mean duration of gavage feeding and oral feeding for the study and control groups was (2.25 \& 19.62) and $(5.83 \& 9.71)$ respectively. Added to that, there was a statistically significant difference between the study and the control group regarding the duration of gavage feeding and oral feeding ( $p=0.001 \& p=0.050$, respectively). This is evident that cycled lighting might enhancement oral feeding.

Table 3: Changes of mean infants' weight in both groups throughout intervention phases (at admission, 10 days and at discharge).

\begin{tabular}{|c|c|c|c|}
\hline Weight & Study $(n=20)$ & Control $(n=20)$ & P-value \\
\hline \multicolumn{3}{|c|}{ Weight at admission } & \multirow{3}{*}{0.447} \\
\hline Mean \pm SD & $2.00 \pm 0.46$ & $2.05 \pm 0.78$ & \\
\hline Range & $1.5-3.0$ & $1.5-4.1$ & \\
\hline \multicolumn{3}{|c|}{ At 10 ${ }^{\text {th }}$ day } & \multirow{3}{*}{$0.017 *$} \\
\hline & & & \\
\hline$\frac{\text { Mean } \pm \text { SD }}{\text { Range }}$ & $\frac{2.45 \pm 0.48}{1.7-3.6}$ & $\frac{2.19 \pm 0.78}{1.5-4.3}$ & \\
\hline \multicolumn{3}{|c|}{ At discharge } & \multirow{3}{*}{0.058} \\
\hline Mean \pm SD & $2.56 \pm 0.47$ & $2.33 \pm 0.80$ & \\
\hline Range & $1.7-3.6$ & $1.3-4.4$ & \\
\hline
\end{tabular}


Table (3): shows the effect of intervention protocol on studied infants' weight throughout specific time parameters (on admission, 10 days and on discharge). There was similarity of mean infants' weight on admission between the study (cycled lighting) and (continuous lighting) control groups $(2.00 \pm 0.46 \& 2.05 \pm 0.78$,
Respectively). After 10 days with the intervention, the infants in study group showed a significantly greater weight gain than those in control (P. 0.017). On discharge, there was no statistically significant difference between two groups. In spite of this, the mean infants' weight in study group was still higher than the control group $(2.56 \pm 0.47 \& 2.33 \pm 0.80$, respectively $)$

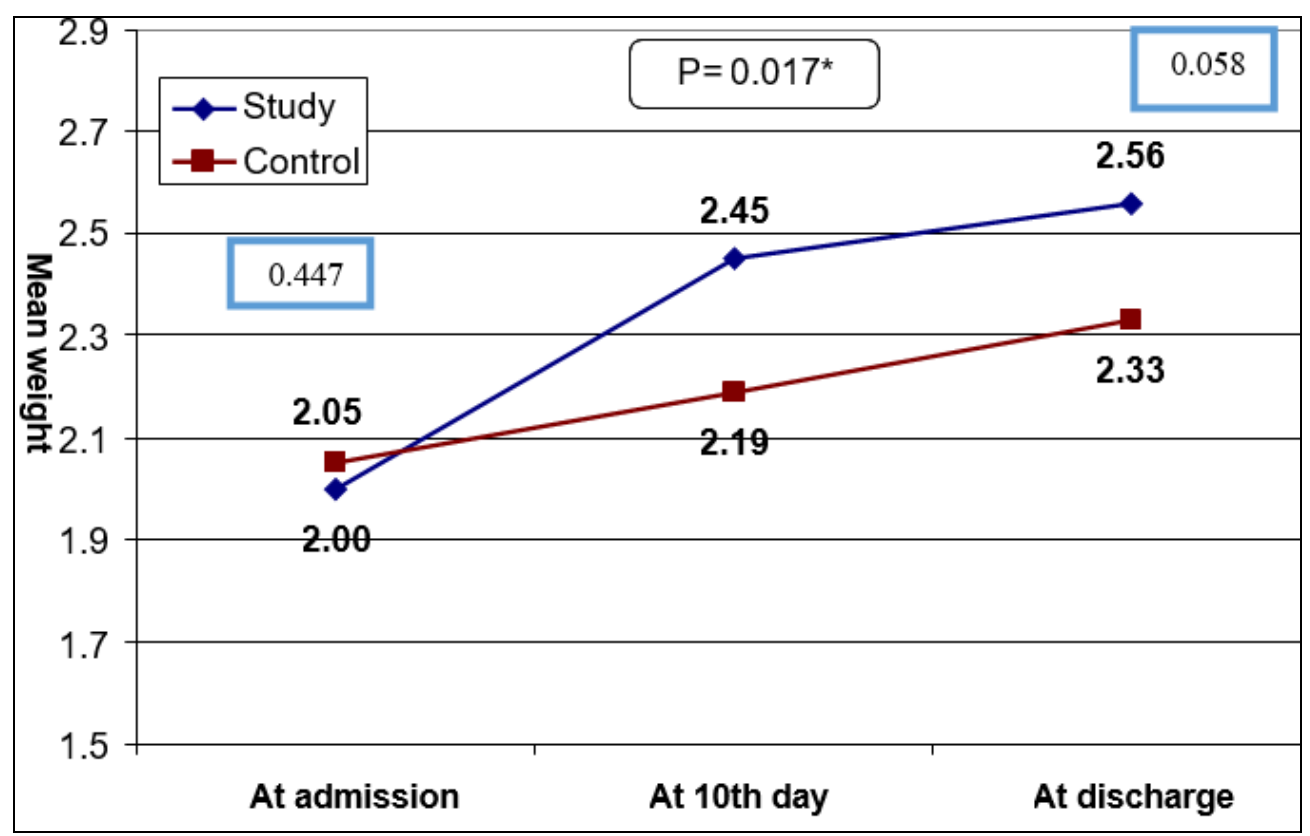

Fig 1: Mean weights' changes of infants in the study and control group throughout intervention phases (at admission, 10 days and at discharge

Table 4: Comparison mean infants' cardiorespiratory function between the two groups throughout intervention phases (at admission, at 10 days and at discharge)

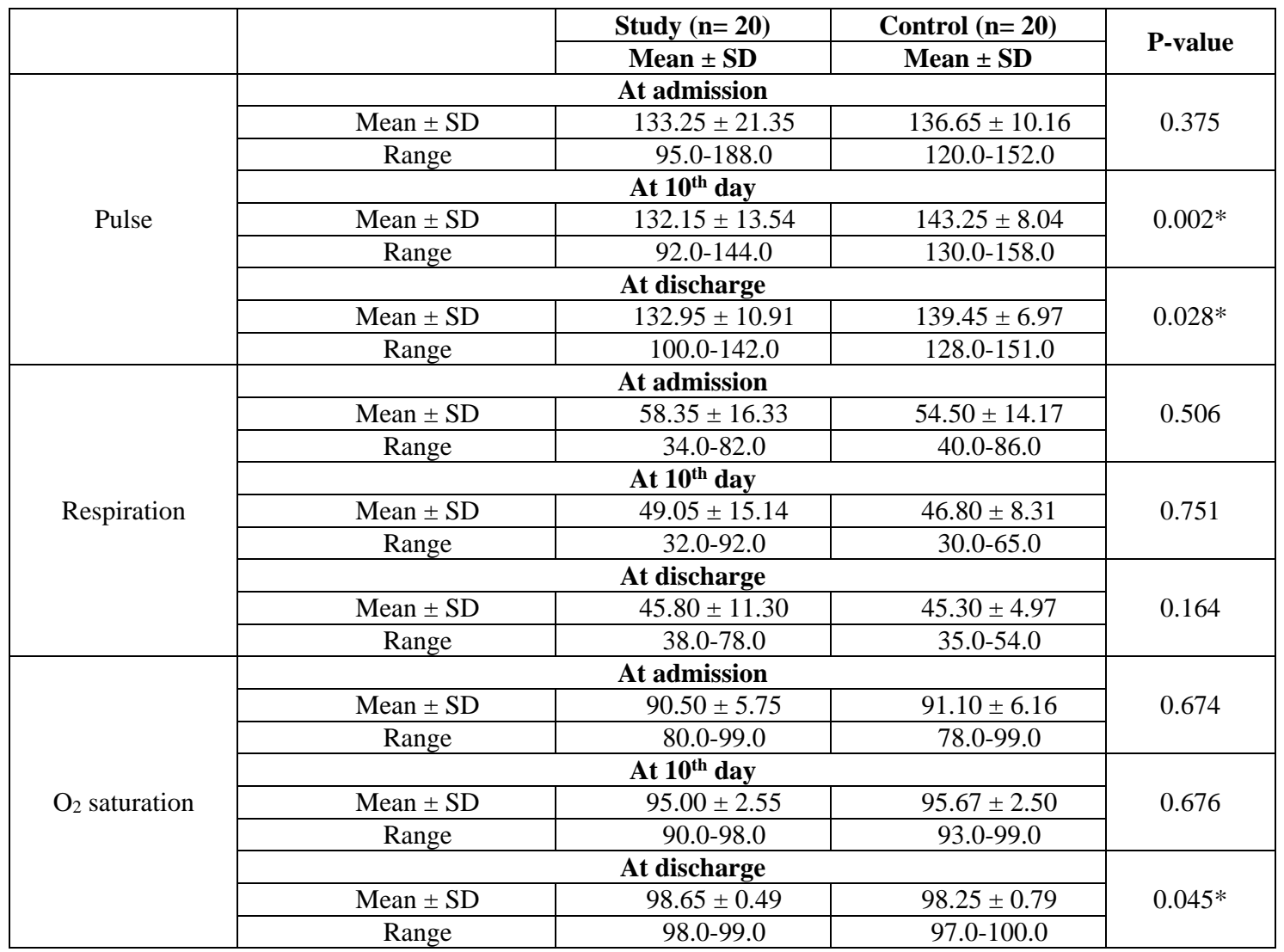

Table (4) compares the pulse, respiration, and $\mathrm{O}_{2}$ saturation for the study (cycled lighting) and control group (continuous lighting) throughout three point of time. At admission, there is no significant differences between the two groups regarding studied three parameters of cardiorespiratory function. Regarding pulse parameter, pulse improvement were revealed in both groups and mean pulse rates at 10 days and at discharge were more stable in the study group (132.15 and 132.95, respectively) compared to 
control group (143.25and 139.45 , respectively) with statistically significant differences $(p<0.002 \& p<0.028)$. This revealed that cycled lighting might have effect on infants' pulse stability and improvement. While, there were no statistically significant differences between two groups for the mean respiration at $10^{\text {th }}$ day and at discharge $(P=0.751 \& P=0.164$, respectively). The comparison of two groups using the mean calculated for $\mathrm{O}_{2}$ saturation levels revealed that there was no significant difference between them at 10 days $(P=0.676)$ but later on discharge the mean $\mathrm{O}_{2}$ saturation for the study group significantly good compared to the control group $(P=0.045)$. This positive finding may also be related to the fact that the light control intervention occurred over longer periods.

Table 5: Duration of hospital stay (days)

\begin{tabular}{|c|c|c|c|}
\hline Hospital stay (days) & Study(n=20) & Control $(\mathbf{n}=\mathbf{2 0})$ & P-value \\
\hline Mean \pm SD & $16.55 \pm 7.71$ & $20.15 \pm 8.95$ & \multirow{2}{*}{0.110} \\
\hline Median (Range) & $14.5(9.0-34.0)$ & $18.0(10.038 .0)$ & \\
\hline
\end{tabular}

Regard to the comparison between both groups regarding duration of hospitalization in NICU, table (5) shows infants in the study group spent 9 to 43 (mean 16.5) days before discharge compared to 10 to 38 (mean 20.1) in the control group, with no statistically significant difference $(P=0.11)$.

\section{Discussion}

The findings of the current study covered three main areas discussed within the following frame of references: First, characteristics of the studied participants. Second, the effect of cycled lighting on infants' weight, cardiorespiratory function and on the duration of hospitalization. Regarding characteristics of the studied infants in in the study (infants exposed to cycled lighting) and control (infants exposed to continuous lighting) groups, results of the current study specified that there were similarities between the two groups regarding sex, mode of delivery and mean of gestational age on admission. Furthermore, there was no significant difference between the two groups regarding weight on admission. In the same line Farahani et al., (2018) ${ }^{[4]}$. Who compare the effects of cycled lighting (CL) and constant lighting on weight gain and the length of stay in NICU among premature neonates. They mentioned that there were no statistically significant differences between two groups regards neonates' route of delivery, gestational age and reason for hospitalization. In addition, Lebel, et al., (2017) ${ }^{[9]}$. Noticed that there was no significant difference between characteristics of the preterm infants randomized to the cycled lighting group and those randomized to the continuous near darkness group with regard to gestational age, weight at admission, type of delivery and respiratory support.

As regards, the effects of CL and constant lighting on studied infants' weight from admission at NICU until discharge. The mean of weight in the study group was not significantly different from the control group at admission. At the 10th day, neonates in both groups started to gain weight. This weight gain can be attributed to the fact that almost all neonates physiologically lose $10 \%-15 \%$ of their birth weight in the 1st week after birth and then start to gain weight in the 2nd week. (Noel-Weiss, et al., 2008) ${ }^{[16]}$. The mean of weight at $10^{\text {th }}$ day in the study group was significantly greater than the control group. In addition, at discharge weight gain is still higher in the study group than control but without significant differences. Of course, the higher weight in the study group can be due to the positive effects of CL. An explanation for this difference between two groups comes from the results of the current study, which evidenced that cycled lighting might enhancement oral feeding. Add to that, Morag and Olsson, (2011) ${ }^{[15]}$. Supported this and mentioned that artificial cyclical lighting could enhance the quality of nutrition, growth and reduce mortality rate among preterm neonates. Moreover, the fact that the establishment of circadian rest-activity patterns and postnatal weight gains among preterm infants is accelerated in Neonatal Intensive Care Units that employ regular light-dark cycles (Ikeda, et al., 2015) ${ }^{[7]}$.

In harmony with the present findings, the two-group randomized controlled trial by Vasquez-Ruiz. et al., (2014) was designed comparing infants in the NICU of Hospital Juarez de México, exposed from birth either to a light/dark (LD) environment $(n=19)$ or to the traditional continuous light $(n=19)$. Light/dark cycle intervention was achieved by placing individual removable helmets over the infant's heads. Body weight gain was analyzed, as the main indicator of stability and the main criteria for discharge. Results showed that infants maintained in an LD cycle gained weight faster than infants in traditional continuous light did $(P>0.01)$. Similarly, Esmaeilizadeh, et al., (2016) ${ }^{[3]}$. Did a research to test the effects of light exposure on weight gain among preterm neonates hospitalized at the NICU Bojnord, Iran. They recruited 60 preterm neonates. The neonates in the first group were exposed to continuous light while in the second group; the neonates were placed in an environment with cyclical lighting until the time of their hospital discharge. They reported that neonates with the simulated cyclical lighting model had greater weight gain. Moreover, cyclical lighting can increase the production of growth related factors, facilitate weight gain and improve health status among neonates. All of the previously mentioned studies assured the importance and good effect of cycled lighting.

A more recent study done by Farrakhan et al., (2018) who compare the effects of $\mathrm{CL}$ and constant lighting on weight gain among premature neonates. In their study CL consisted of $12 \mathrm{~h}$ of normal NICU lighting and $12 \mathrm{~h}$ of reduced lighting by using a sheet of acrylic glass (commercially known as Plexiglas) covered by a dimming cotton cover. The results found that there was no significantly difference between two groups respecting neonates' weight in the first 8 th days $(P=0.857)$. While weight mean in days $9-15$ in the intervention group was significantly greater than the control group $(P<0.001)$. Therefore, the relatively old and recent researches supported the current study findings and confirmed the importance of the application of Cyclical lighting, meaning natural day/night-based lighting on facilitate weight gain. The results of other studies in this area are in contrary to the current study findings. For instance, A quasi-experimental study was performed on 38 preterm neonates, who were randomly allocated to cyclical lighting (experimental) or continuous lighting (control) groups for five days had no significant difference between the groups neither before nor after the study intervention regarding the pattern of weight gain (Reyhani and Sanadgol, 2016) ${ }^{[18]}$. An explanation for this contradiction is the fact that day-night synchronized cyclical lighting does not significantly affect neonates' weight gain during the first days of the intervention (Rivkees et al., 2004) ${ }^{[19]}$.

Another study also was carried out on preterm infants admitted to a neonatal intensive care unit. The aim was to compare the weight gain between preterm infants exposed to $12 \mathrm{~h}$ cyclical lighting (CL) and those exposed to a continuously dim environment (both started in hospital and continued after hospital discharge). It showed no significant difference between the effects of CL and dim lighting on weight gain of premature infants (Mirmiran, et al., 2003). This is contrary to present study findings may be due to the differences in lighting intensity. In current study, neonates of the control group were exposed to continuous lighting while neonates in their control group placed with dim lighting. Seemingly, dim lighting is less stressful for neonates than continuous lighting, resulting in insignificant difference between the groups in Mirmiran, et al., study.

The effect of $\mathrm{CL}$ on studied parameters of cardiorespiratory function has been explored in the present study. Regarding pulse parameter, the findings point to cycled lighting might have effect 
on infants' pulse stability and improvement. Add to that, the comparison of two groups regarding $\mathrm{O}_{2}$ saturation levels revealed that there was no significant difference between them at $10^{\text {th }}$ day but later on discharge the mean $\mathrm{O}_{2}$ saturation for the study group significantly good compared to the control group. Therefore, a controlled intervention (cycled lighting) may have systematically favored physiological stability with a continuous bright light comparison group. In line with these findings, a study proved that CL caused significant positive physiological changes compared with constant lighting and found CL effective in promoting heart rate stability (Reyhani and Sanadgol 2016) ${ }^{[18]}$. Too, a randomized study with intervention exposure time from birth until hospital discharge observed improvement in oxygen saturation and in the rhythmicity of the heart rate in infants exposed to cycled lighting over continuous bright light (Vásquez-Ruiz, et al., 2014) ${ }^{[20]}$. In addition, Morag and Ohlsson (2013) stated that bright light to be associated with a significantly greater amount of active rapid eye movement (REM) sleep, which was regarded as a relatively destabilized state with fluctuations in heart rate, blood pressure and oxygen saturation.

In contrast, Lebel, et al., (2017) ${ }^{[9]}$. Study found no statistically significant differences in physiological stability in preterm infants born between 28 and 32 weeks when exposed to cycle lighting and continuous near darkness. In addition, infants were physiologically stable throughout the study in both intervention groups. This discrepancy might be attributed to the differences in neonates' gestational age and the cycled protocols used in these two studies. Another explanation for the lack of differences in their study that these light-controlled interventions (cycled lighting and continuous near darkness) are safe for physiologic stability of premature infants.

The present study findings revealed that the study groups did not differ significantly from each other regarding infants' NICU stay duration. Similarly, a recent study compared the effects of cycled lighting (CL) and constant lighting on the length of stay in NICU among premature neonates found that no significant difference was observed between the two groups (Farahani, et al., 2018) ${ }^{[4]}$. While a study illustrated that significantly short hospital stay among the neonates treated with the simulated cyclical lighting model compared with the neonates who were exposed to continuous lighting (Esmaeilizadeh, et al., (2016) ${ }^{[3]}$. A probable explanation for such contrary findings may be the differences in the gestational ages and intervention protocol used of these two studies. Besides, the length of neonates' stay in the hospital is a good indicator for assessing the quality of health-care services (Atienza, et al., 2008). Finally, this study has several important findings that can be considered as a basis for more comprehensive studies on the effects of cyclical lighting on preterm neonates in the Upper Egypt. As with any research, there are certain limitations of the study firstly, the sample size was relatively small, but it is comparable to previous studies that measured the impact of lighting control on preterm infants. Secondly, the study setting was a single NICU. Therefore, the findings should interpreted and generalized with caution.

\section{Conclusion}

The study concluded that implementation of cycled lighting protocol led to statistically significant improvements in the pattern of weight gain, pulse stability and $\mathrm{O} 2$ saturation in the preterm neonates. Investigators have observed the motivating conclusion during the study that there was no significant difference in the mean of hospital stay between preterm infants exposed to cyclic lighting and those exposed to continuous light.

\section{Recommendations}

\section{On the light of the study findings, it is recommended that:}

- Nurses in NICU can use the findings to improve neonatal outcomes among premature neonates hospitalized in NICUs.

- Future multicenter researches needed to provide strong evidence respecting the effects of $\mathrm{CL}$ on preterm health and development.
- Further researches are still needed to examine effects of CL on both physiologic and circadian rhythm outcomes.

\section{References}

1. Alizadeh P, Goudarzi Z, Shariat M, Nariman S. Effect of sunflower oil massage on length of hospital stay in preterm infants in selected hospitals of Tehran University of Medical Sciences in 2012. J Alborz Univ. Med. Sci. 2013; 2:59-66.

2. Atienza N, García-Heras J, Muñoz-Pichardo JM, Villa R. An application of mixture distributions in modelization of length of hospital stay. Stat Med. 2008; 27:1403-20.

3. Esmaeilizadeh M, Shoja M, Shoja E, Shoja M, Nejat H, Oudi D. Comparing the effects of continuous and cyclical lightings on weight gain and length of hospital stay among preterm neonates. Modern Care Journal, 2016, 13(1).

4. Farahani EA, Nourian M, Ahmadi F, Kazemian M. Comparing the effects of cycled and constant lighting on weight gain and length of stay in neonatal intensive care unit among premature neonates: A two-Group randomized controlled clinical trial. Nurs Midwifery Stud. Journal. 2018; 7:93-9.

5. Guyer C, Huber R, Fontijn J. Cycled light exposure reduces fussing and crying in very preterm infants. Pediatrics. 2012; 130(1):145-151.

6. Hunt KN. the NICU: Environmental Effects of the Neonatal Intensive Care Unit on Infants and Caregivers. Research [Accessed on: January 12, 2016]. Available at: http://opensiuc.lib.siu.edu/cgi/viewcontent.cgi?article). 2011, 71.

7. Ikeda T, Ishikawa, H, Shimizu K, Asakawa K, Goseki T. Pupillary size and light reflex in premature infants. NeuroOphthalmol. 2015; 39:175-178.

8. Klock P, Erdmann AL. Caring for newborns in a NICU: Dealing with the fragility of living/surviving in the light of complexity. Rev Esc Enferm USP. 2012; 46:45-51.

9. Lebel V, Aita M, Johnston C, Héon M. Effects of Cycled Lighting Versus Continuous Near Darkness on Physiological Stability and Motor Activity Level in Preterm Infants. Advances in Neonatal Care. 2017; 17(4):282-291.

10. Lee J, Bang K. The effects of Kangaroo care on maternal selfesteem and premature infants' physiological stability. Korean J Woman Health Nurs. 2011; 17(5):454-462.

11. Martin RJ, Fanaroff AA, Walsh MC. Faroff and Martin's Neonatal-Perinatal Medicine: Diseases of the Fetus and Infant. Philadelphia: Elsevier Health Science. 2014.

12. Mirmiran M, Baldwin RB, Ariagno RL. Circadian and sleep development in preterm infants occurs independently from the influences of environmental lighting. Pediatr Res. 2003; 53:933-8.

13. Morag I, Ohlsson A. Cycled light in the intensive care unit for the preterm and low birth weight infants. Cochrane Database Syst Rev. 2013; (8):6982.

14. Morag I, Ohlsson A. Cycled light in the intensive care unit for preterm and low birth weight infants. Cochrane Database of Systematic Reviews. 2016; 8:6982.

15. Morag I, Ohlsson A. Cycled light in the intensive care unit for preterm and low birth weight infants. Cochrane Database Syst Rev. 006982.doi: 10.1002/14651858.CD006982, 2011, (1).

16. Noel-Weiss J, Courant G, Woodend AK. Physiological weight loss in the breastfed neonate: a systematic review. Open Med. 2008; 2(4):99-110.

17. Peng N, Bachman $\mathrm{H}$, Jenkins $\mathrm{R}$, Chen $\mathrm{CH}$, et al. Relationships between environmental stressors and stress biobehavioral responses of preterm infants in NICU. J Perinat Neonatal Nurs. 2009; 23(4):363-71.

18. Reyhani T, Sanadgol V. The Effects of Creating an Artificial Night on the Pattern of Weight Gain Among Preterm Neonates, Mod Care J. 2016; 13(1):8843.

19. Rivkees SA, Mayes L, Jacobs H, Gross I. Rest-activity patterns of premature infants are regulated by cycled lighting. Pediatrics. 2004; 113(4):833-839. 
20. Vásquez-Ruiz S, Maya-Barrios JA, Torres-Narváez P, VegaMartínez BR, Rojas-Granados A, Escobar C et al. A light/dark cycle in the NICU accelerates body weight gain and shortens time to discharge in preterm infants. Early Hum Dev. 2014; 90:535-40.

21. Verklan MT, Walden M. Core Curriculum for Neonatal Intensive Care Nursing. $5^{\text {th }}$ ed. St. Louis, Missouri: Elsevier Health Science, 2014

22. World Health Organization (WHO) Preterm Birth; Available from:

http://www.who.int/mediacentre/factsheets/fs363/enwere 240, 2017. 